\title{
Hematopoyesis extramedular: ¿mecanismo compensador o síndrome clínico? Descripción de un caso y revisión bibliográfica
}

\author{
J. ROSADA, M. BINDI, M. PINELLI, C. PANDOLFO, G. CASSETTI, \\ M. CASTIGLIONI \\ Unitá Operativa Medicina Interna IV - Ospedale Cisanello, Azienda Ospedaliera. \\ Universitaria Pisana, Italia
}

\begin{abstract}
RESUMEN
La hematopoyesis extramedular (HEM) es clásicamente considerada un mecanismo compensador en pacientes con anemia crónica. Hígado, bazo y ganglios linfáticos son frecuentemente implicados. Sin embargo la HEM puede también desarrollarse, de manera generalmente asintomática, en otras localizaciones como timo, riñón, retroperitoneo, área paravertebral del tórax, pulmón, intestino y otras. Raramente es sintomática (HEMS), presentándose con una gran variedad de cuadros clínicos con evolución a veces fatal. Esta forma es subvalorada y poco conocida. Su reconocimiento como entidad clínica permite un diagnóstico precoz evitando, cuando es posible, una evolución letal. Los autores describen un caso donde fueron encontradas células de la médula ósea en el análisis del líquido cefaloraquídeo (LCR) de un paciente con linfoma no Hodg$\operatorname{kin}(\mathrm{LNH})$.
\end{abstract}

PALABRAS CLAVE: Hematopoyesis extramedular. Líquido cefaloraquídeo. Linfoma no Hodgkin.
EXTRAMEDULLARY HEMATOPOIESIS: COMPENSATORY MECHANISM OR CLINIC SYNDROME? CASE REPORT AND REVIEW OF LITERATURE

\section{ABSTRACT}

Extramedullary hematopoiesis (EMH) is a compensatory mechanism occurring in patients with chronic anemia. Liver, spleen, and lymph nodes are frequently involved. However, EMH may also develop in several sites such as thymus, kidneys, retroperitoneum, paravertebral areas of the thorax, lungs, bowel and others. Rarely symptomatic, it often shows a variety of clinical features. This condition, frequently, may be fatal. A correct early diagnosis of EHM might avoid, if possible, a bad prognosis. The Authors report a case where bone marrow cells were identified in centrifuge cerebrospinal fluid of a patient suffering from non-Hodgkin lymphoma.

KEY WORDS: Extramedullary hematopoiesis. Cerebrospinal fluid. Non-Hodgkin lymphoma.

Rosada J, Bindi M, Pinelli M, Pandolfo C, Cassetti G, Castiglioni M. Hematopoyesis extramedular: ¿mecanismo compensador o síndrome clínico? Descripción de un caso y revisión bibliográfica. An Med Interna (Madrid) 2007; 24: 77-80.

\section{INTRODUCCIÓN}

La presencia de células hematopoyéticas progenitoras con localización extramedular es característica de la etapa de vida intra-uterina. Durante el segundo trimestre de gestación el hígado se convierte en la principal fuente productora de glóbulos rojos, aunque si una cierta cantidad de hematíes se produce aún en el bazo y en los ganglios linfáticos. A partir de los ùltimos meses de gestación y hasta los 5 años de edad, los eritrocitos, granulocitos y monocitos se producen exclusivamente en la médula ósea de casi todos los huesos. Después de los 20 años los componentes de las tres principales líneas hematopoyéticas se forman en el tejido medular de los huesos planos. También en estos huesos la hematopoyesis disminuye con el avanzar de la edad (1). Se describe un caso de HEM con localización meníngea y se revisa la literatura a propósito.

\section{CASO APORTADO}

Varón italiano de 64 años ingresa en marzo del 2005 por intensa cefalea, fiebre continua y pancitopenia. En la historia resulta padecer de LNH di tipo B grado II (Real) ya tratado con quemioterapia según el esquema CHOP-R y sucesivamente con altas dosis de ciclofosfamida con el objetivo de movilizar las células estaminales en previsión de autotrasplante, por otra parte, nunca efectuado. Una reciente TAC total body describía una remisión de la enfermedad linfomatosa mientras la biopsia osteomedular evidencia una mielodisplasia secundaria en ausencia de infiltración neoplásica.

A su ingreso en el hospital en la exploración física se aprecia fiebre de $39{ }^{\circ} \mathrm{C}$, palidez cutaneomucosa y esplenomegalia, PA 110/70 $\mathrm{mmHg}$, frecuencia cardiaca 128 latidos por minuto. El resto de la exploración física inicial es compatible con la normalidad.

Trabajo aceptado: 20 de septiembre de 2006 
Exploraciones complementarias. Los primeros análisis (Tabla I) muestran, entre otras cosas, pancitopenia con neutropenia de 830/ul. Se efectuaban exámenes virológicos (negativos) y hemocultivos seriados. Una TAC de cráneo no mostraba lesiones focales agudas ni signos de ipertensión cerebral. El paciente se trata con terapia de sostén, factores de crecimiento granulocitario, antimicóticos y antibióticos de amplio espectro de acción, con remisión de cuadro hematológico (Tabla I) y resolución de la fiebre. Por el persistir de la cefalea y la aparición sucesiva de signos meníngeos se efectuaba una punción lumbar diagnóstica. El análisis microscópico del LCR muestra un incremento del número total de células, constituidas por un gran número de neutrófilos, megacariocitos y células mieloides con maduración intermedia; cuadro representati-

\section{TABLA I}

\begin{tabular}{|c|c|c|}
\hline $\begin{array}{l}\text { Algunos exámenes } \\
\text { hematoquímicos valor normal }\end{array}$ & Ingreso & 10 días después \\
\hline Ematocrito (vn. 40-54) & $26,90 \%$ & $38,4 \%$ \\
\hline MCV (vn. 82-98) & $76,9 \mathrm{fL}$ & $80 \mathrm{fL}$ \\
\hline MCH/MCHC(27-31/ 32-36) & $26,9 \mathrm{pg} / 34,9 \mathrm{~g} / \mathrm{dl}$ & $27 \mathrm{pg} / 33,9 \mathrm{~g} / \mathrm{dl}$ \\
\hline Plaquetas (vn. 130-350) & $44,0 \times 10^{3} / u l$ & $80 \times 10^{3} / \mathrm{ul}$ \\
\hline GB (vn. 4,5-10.0) & $1,11 \times 10^{6} / \mathrm{ul}$ & $6,71 \times 10^{6} / u l$ \\
\hline PMN (40-74/ 1,9-8) & $75 \% / 0,83 \times 10^{3} / u l$ & $94,7 \%$ \\
\hline Linfocitos (vn. 19-48) & $2,7 \%$ & $2,7 \%$ \\
\hline Monocitos (vn. 3,4 -9) & $1,9 \%$ & $1,9 \%$ \\
\hline Eosinófilos(vn. 0,0-5,0) & $0 \%$ & $0 \%$ \\
\hline Basófilos (vn. 0.0- 0.5) & $0 \%$ & $0,3 \%$ \\
\hline Reticulocitos: (vn. 0,5-2,5) & $1,8 \%$ & $1,8 \%$ \\
\hline Ferritina (vn. 26-306) & $750 \mathrm{ug} / \mathrm{ml}$ & 594 ug/ml \\
\hline INR (vn.0,8-1,3) & 1,48 & 1,48 \\
\hline Vitamina $B_{12}(179-1162)$ & $1.111 \mathrm{ug} / \mathrm{L}$ & - \\
\hline Ácido fólico (vn. 2,7-34) & $7 \mathrm{ng} / \mathrm{ml}$ & - \\
\hline Bilirrubina t. $(0,2-1,2)$ & $1,3 \mathrm{mg} / \mathrm{dl}$ & $1,3 \mathrm{mg} / \mathrm{dl}$ \\
\hline Bilirrubina directa $(0,1-0,4)$ & $0,1 \mathrm{mg} / \mathrm{dl}$ & $0,22 \mathrm{mg} / \mathrm{dl}$ \\
\hline AST (vn. 5-45) & $11 \mathrm{mg} / \mathrm{dl}$ & $17 \mathrm{mg} / \mathrm{dl}$ \\
\hline ALT (vn. 5-45) & $15 \mathrm{mg} / \mathrm{dl}$ & $17 \mathrm{mg} / \mathrm{dl}$ \\
\hline F. alcalina (vn. 90-260) & $90 \mathrm{U} / \mathrm{L}$ & $155 \mathrm{U} / \mathrm{L}$ \\
\hline LDH (vn. 230-460) & $186 \mathrm{U} / \mathrm{L}$ & $186 \mathrm{U} / \mathrm{L}$ \\
\hline PCR (vn. 0-5) & $25,9 \mathrm{mg} / \mathrm{L}$ & $3,05 \mathrm{mg} / \mathrm{L}$ \\
\hline Proteinas t. (vn. 6-8) & $5,8 \mathrm{~g} / \mathrm{dl}$ & $5,9 \mathrm{~g} / \mathrm{dl} /$ \\
\hline Albúmina (vn. 3,7-5,3) & $3,1 \mathrm{~g} / \mathrm{dl}$ & $3,8 \mathrm{~g} / \mathrm{dl}$ \\
\hline Creatinina (vn. 0,4-1,4) & $0,80 \mathrm{mg} / \mathrm{dl}$ & $0,7 \mathrm{mg} / \mathrm{dl}$ \\
\hline Clicemia (vn. 60-110) & $139 \mathrm{mg} / \mathrm{dl}$ & $137 \mathrm{mg} / \mathrm{dl}$ \\
\hline $\mathrm{Na}^{+}(\mathrm{vn} .136-146)$ & $127 \mathrm{mEq} / \mathrm{L}$ & $143 \mathrm{mEq} / \mathrm{L}$ \\
\hline $\mathrm{K}^{+}(\mathrm{vn} .3,5-5,1)$ & $4 \mathrm{mEq} / \mathrm{L}$ & $4,4 \mathrm{mEq} / \mathrm{L}$ \\
\hline $\mathrm{Ca}$ (vn. 8,2-10,2) & $7,1 \mathrm{mg} / \mathrm{dl}$ & $8,4 \mathrm{mg} / \mathrm{dl}$ \\
\hline$M g(v n 1,7-2,45)$ & $2,4 \mathrm{mg} / \mathrm{dl}$ & $2 \mathrm{mg} / \mathrm{dl}$ \\
\hline pH arterioso (vn. 7,35-7,45) & 7,44 & 7,47 \\
\hline $\mathrm{PO}_{2}($ vn. 80-110) & $86,6 \mathrm{mmHg}$ & $92,2 \mathrm{mmHg}$ \\
\hline $\mathrm{PCO}_{2}$ (vn. 35-45) & $27,7 \mathrm{mmHg}$ & $36,5 \mathrm{mmHg}$ \\
\hline $\mathrm{HCO}_{3}(25)$ & $19,1 \mathrm{mmol} / \mathrm{l}$ & $26,2 \mathrm{mmol} / \mathrm{l}$ \\
\hline $\mathrm{SO}_{2 \text { inaria }}$ & $96,7 \%$ & $97,8 \%$ \\
\hline Anticuerpos antifosfolípidos & negativos & - \\
\hline Anti HIV 1-2 & negativos & - \\
\hline Antígenes virales $L C R$ & negativos & negativos \\
\hline Cultivos del LCR & negativos & negativos \\
\hline
\end{tabular}

vo de una población medular. El análisis citológico inmunohistoquímico se presentaba compatible con metaplasia mieloide: factor VIII y mieloperoxidasa positivos en los elementos morfológicamente compatibles. El análisis repetido del LCR en comparación con la sangre periférica y el hecho que ninguna raquicéntesis fuera traumática o de difícil ejecución excluye la contaminación. Mientras tanto resulta positivo el hemocultivo a Sterotrophomonas maltophilia sensible al tratamiento antibiótico en acto. Dos semanas después del ingreso las condiciones neurológicas del paciente empeoran, entra en coma cerebral y fallece.

\section{DISCUSIÓN}

La HEM en el adulto es infrecuente y en general considerada un mecanismo compensador en pacientes con anemia crónica. Esta condición puede presentarse en curso de hemoglobinopatías, anemias hemolíticas, leucemias, enfermedades mieloproliferativas, linfomas o asociada a una pancitopenia yatrogénica. Raramente acompañando anemias carenciales (2), precedendo la manifestación clinica de la enfermedad hematológica a la base (3) o en ausencia de alteración hematológica asociada (4). Bazo, hígado y ganglios linfáticos son frecuentemente implicados, más raramente pulmón, timo, riñón, aparato gastrointestinal y urinario, retroperitoneo, área paravertebral del tórax, SNC y otros. Como suele ser asintomática la mayor parte de las veces es un hallazgo post-mortem.

Cuando la HEM es sintomática (HEMS) no debería ser considerada un mecanismo compensador sino una verdadera entidad clínica que se presenta, según el órgano o aparato interesado, con una gran variedad de cuadros clínicos. Para facilitar la descripción de algunos de estos nosotros preferimos agruparlos en 6 tipos: a) la HEMS que complica el SNC y periférico, uno de los más frecuentes en esta rara patología, que puede presentarse como cefalea intratable (5-7), afectactación cerebelar (8), afasia (7), hemiparesis (7), pérdida del control esfintérico $(6,7)$, otros signos de compresión medular (9-12), una banal ciatalgia (13), un exoftalmo monolateral(7), una hemorragia subdural (14) o paraparesis-paraplejía $(6,15)$; b) la HEMS torácica puede agredir los pulmones y la pleura dando lugar a fibrosis pulmonar (16), derrame pleuritico mono o bilateral (17-19), hemotórax $(20,21)$ a veces fatales (22), se puede presentar como una masa mediastinal o intratorácica de difícil interpretación (23) o infiltrar el corazón presentándose como precordialgia, disnea cardiaca (24), tamponamiento (24-26) o masa intraatrial $(27,28)$ (la afectación miocárdica en particular la asociación con IMA es al contrario discutible); c) en la HEMS abdominal es frecuente la infiltración peritoneal como ascitis severa (19), incluso refractaria (29) o como un tejido proliferante que determina, en esta última forma, problemas en el diagnóstico diferencial de la carcinomatosis o del mesotelioma peritoneal (30), cuando su localización es perirenal o retroperitoneal se asocia a cuadros de uropatía obstructiva, infiltración tubulointersticial y urolitiasis, síndrome nefrótico y glomerulonefritis $(31,32)$, insuficiencia renal aguda (33) o puede manifestarse, también aquí, como una masa de dudoso significado (34); d) en la presentación intestinal, además de las hemorragias (35), se reportan casos de estreñimiento importante (36), de oclusión o abdomen agudo quirúrgico (37); e) la HEMS cutánea, de peor pronóstico, puede presentarse sola o acompañando las otras formas $(26,38)$ y es caracterizada por un cuadro heterogéneo: 
nódulos, lesiones papulares rojo-violetas (38), eritema papular o difuso, lesiones ulceradas, placas eritematosas, ampollas $(39,40)$; y f) otras localizaciones menos reportadas son tiroides $(3,41)$ como un nódulo único o como bocio multinodular, suprarrenal (42), testículo (38), próstata (43), ovario (44), endometrio (45) y oído medio (46) (todos los tejidos, órganos o sistemas pueden ser interesados).

El tratamiento de la HEMS es muy discutido y no existe un acuerdo entre los diferentes autores. En los casos no fatales registrados algunos se resuelven espontaneamente (21), otros con un intenso régimen de trasfusiones $(11,12,15)$, con radioterapia $(7,31)$, cirugía o con combinaciones $(47,48)$ de estos tratamientos según la localización y la modalidad de presenta- ción. La HEM es una patología infrecuente, poco conocida, con frecuencia de tipo compensador a una hemopoyesis medular alterada. En nuestro caso la infiltración meningoencefálico de la HEM en vez de comportarse como un mecanismo compensador de la hematopoyesis por el daño medular postquemioterápico, desarrolló un cuadro sindrómico neurológico con evolución letal. Los cuadros clinicos de presentación son numerosos y con evoluciones diversas. El conocimiento de esta patología nos ayuda por un lado, a no disminuir la importancia de una sintomatología no precisa que si se diagnostica precozmente, puede ser tratada y por otro lado a evitar, cuando es posible, una evolución desfavorable.

\section{Bibliografía}

1. Guyton AC, Hall JE. Globuli rossi, anemia e policitemia. In: EdiSES s.r.1., editors. Fisiologia Medica. 9th ed. Cità di Castello (PG): Sograte s.r.l.; 1999, p. 431-43.

2. Fielding JR, Owens M, Naimark A. Intrathoracic extramedullary hematopoiesis secondary to B12 and folate deficiency: CT appearance. J Comput Assist Tomogr. 1991; 15: 308-10.

3. Leoni F, Fabbri R, et al. Extramedullary haematopoiesis in the thyroid multinodular goitre preceding clinical evidence of agnogenic myeloid metaplasia. Histopathology 1996; 28: 559-61.

4. Bickhardt J, Schheithauer J, Matthiessen W. (Extramedullary hematopoiesis-a differential diagnosis of intrathoracic paravertebral tumors Pneumologie 2004; 58: 660-5.

5. Avvildiz O, Muftuoglu E, et al. Intracranial meningeal extramedullary hematopoiesis inducing serious headache in a patient with idiopathic myelofibrosis. J Pediatr Hematol Oncol 2004; 26: 28-9.

6. Tan TC, Tsao J, Cheung FC. Extramedullary haemopoisis in thalassemia intermedia presenting as paraplegia. J Clin Neurosci 2002; 9: 7215.

7. Landolfi R, et al. Meningeal hematopoiesis causino exophthalmus and hemiparesis in myelofibrosis: effect of radiotherapy. A case report. Cancer 1988; 62: 2346-9.

8. Chishti MK, Bannister CM. Foci of extramedullary haemopoiesis in a cerebellar haemangioblastoma. Br J Neurosurg 1992; 6:157-62.

9. Mutijima E, Hmissa S, Ziadi S, et al. (Cord compression secondary to extramedullary hematopoiesis in a patient with beta-thalassaemia). Rev Med Liege 2005; 60: 41-4.

10. Aliberti B, Patrikiou A, Terentiou A, Frangatou S, Papadimitriou A. Spinal cord compression due to extramedullary haematopoiesis in two patients with thalassaemia: Complete regression with blood transfusion therapy. J Neurol 2001; 248: 18-22.

11. Lee AC, Chiu W, et al. Hypertransfusion for spinal cord compression secondary to extramedullary hematopoiesis. Pediatr Hematol Oncol 1996; 13: 89-94.

12. Stahl SM, Ellinger G, Baringer JR. Progressive myelopathy due to extramedullary hematopoiesis: Case report and review of the literature. Ann Neurol 1979; 5: 485-9.

13. Carazo ER, Herrera RO, de Fuentes TM, Rull JP, et al. Presacral extramedullary haematopoiesis with involvement of the sciatic nerve. Eur Radiol 1999; 9: 1404-6.

14. Brown JA, et al. Subdural hemorrhage secondary to extramedullary ematopoiesis in postpolicythemic myeloid metaplasy. Neurosurgery 1984; 14: 588-91.

15. Singounas EG, et al. Paraplegia in a pregnant thalassemic woman due to extramedullary hematopoiesis: Successful management with transfusions. Surg Neurol 1991; 36: 210-5.

16. Asakura S, Colby TV. Agnogenic myeloid metaplasia with extramedullary hematopoiesis and fibrosis in the lung. Report of two cases. Chest 1994; 105: 1866-8.

17. Ibabao J, Kassapidis S, et al. Bilateral pleural effusions in a beta-thalassemia intermedia patient with posterior mediastinal extramedullary hematopoietic masses. Hemoglobin 1999; 23: 249-53.

18. Taher A, et al. Extramedullary hematopoiesis in patient with beta-thalassemia intermedia manifesting as symtomatic pleural effusion. Hemoglobin 2001; 25: 363-8.

19. Oren I, Goldman A, Haddad N, Azzam Z, et al. Ascites and pleural effusion secondary to extramedullary hematopoiesis. Am J Med Sci 1999; $318: 286-8$

20. Xiros N, Economopoulos T, et al. Massive hemothorax due to intrathoracic extramedullary hematopoiesis in a patient with hereditary spherocytosis. Ann Hematol 2001; 80: 38-40.

21. Chu KA, Lai RS, et al. Intrathoracic extramedullary haematopoiesis complicated by massive haemothorax in alpha-thalassaemia. Thorax 1999; 54: 466-8

22. Chute DJ, Fowler DR. Fatal hemothorax due to rupture of an intrathoracic extramedullary hematopoietic nodule. Am J Forensic Med Pathol 2004; 25: 74-7.

23. Castelli R, Grazia dei G, Karini M, Cappellini MD. Intrathoracic masses due to exramedullary hematopoisis. Am J Med Sci 2004; 328: 299-308.

24. Mani S, Duffy TP. Pericardial tamponade in chronic myelomonocytic leukemia. Chest 1995; 107: 1184.

25. Imam TH, Doll DC. Acute cardiac tamponade associated with pericardial extramedullary hematopoiesis in agnogenic myeloid mataplasia. Acta Haematol 1997; 98: 42-3.

26. Shih LY, Lin FC, Kuo TT. Cutaneos and pericardial extramedullary hematopoiesis with cardiac tamponade in chronic myeloid leukemia. Am J Clin Pathol 1988; 89: 693-7.

27. Freeman RK, Bethel KJ, et al. Extramedullary hematopoiesis presenting as an intraatriale mass in a patient with chronic myelogenous leukaemia. J Thorac Cardiovasc Surg 1995; 110: 552-4.

28. Burke AP, Virmani R. Cardiac myxoma. A clinicopathologic study. Am J Clin Pathol 1993; 100: 671-80.

29. Jacobs P, Wood L, Robson S. Refractory ascites in the chronic myeloproliferative syndrome: A case report. Am J Hematol 1991; 37: 128-9.

30. Scott WW Jr, Fishman EK. Extramedullary hematopoiesis mimicking the appearance of carcinomatosis or peritoneal mesothelioma: Computed tomography demonstration. Gastrointest Radiol 1990; 15: 82-3.

31. Intragumtornchai $\mathrm{T}$, Kasantikul $\mathrm{V}$, et al. Obstructive uropathy due to extramedullary haematopoiesis in beta thalassaemia/haemoglobin E. Postgrad Med J 1993; 69: 75-7.

32. Liu TT, Chen JB, Chen WJ, et al. Idiopathic myelofibrosis associated with renal extramedullary hematopoisis and nephrotic sindrome: Case report. Chang Gung Med J 2000; 23: 169-74.

33. Holt SG, Field P, Carmichael P, et al. Extramedullary haematopoiesis in the renal parenchyma as a cause of acute renal failure in myelofibrosis. Nephrol Dial Transplant 1995; 10: 1438-40.

34. Sharma AK. Tumefactive extramedullarary hematopoiesis of the kidney in a patient with idiopathic thrombocytopenic purpura. Am J Roentgenol 1996; 167: 795-6.

35. Schreibman D, et al. Small intestinal myeloid metaplasia. Jama 1988 ; 259: 2580-2 
36. Solomon D,Goodman H, Jacobs P. Case report: rectal stenosis due to extramedullary haemopoiesis-radiological features. Clin Radiol 1994; 49: 726-8.

37. Payan HM, et al. Acute surgical abdomen and myelofibrosis. Mod Pathol 1989; 2: 270-2

38. Ruberto E, Paz R, et al. (Idiopathic myelofibrosis with extramedullary hematopoiesis foci in the skin and testicles. Report of a case) Sangre (Barc) 1995; 40: 157-60.

39. Loewy G, Mathew A, Distenfeld A. Skin manifestation of agnogenic myeloid metaplasia. Am J Hematol 1994; 45: 167-70.

40. Patel BM, Su WP, Perniciaro C, Gertz MA. Cutaneous extramedullary hematopoiesis. J Am Acad Dermatol 1995; 32 (5 Pt 1): 805-7.

41. Lazzi S, Als C, Laissue J, et al. Extensive extramedullary hematopoiesis in a thyroid nodule. Mod Pathol 1996; 9: 1062-5.

42. Calhoun SK, Bergman K, et al. Extramedullary hematopoiesis in achild with hereditary spherocytosis: An uncommon cause of an adrenal mass. Peditr Radiol 2001; 31: 879-81.

43. Ruberto E, Paz R, at al. (Idiopathic myelofibrosis with extramedullary hematopoiesis foci in the skin and testicles. Report of a case) Sangre (Barc) 1995; 40: 157-60.

44. Humphrey PA, Vollmer RT. Extramedullary hematopoiesis in the prostate. Am J Surg Pathol 1991; 15: 486-90.

45. Huang CW, Hsueh S, Chang MY. Agnogenic myeloid mataplasia in an ovarian steroid cell tumor with virilization: A case report. J Reprod Med 2004; 49: 765-8.

46. Sirgi KE, Swanson PE, Gersell DJ. Extramedullary hematopoiesis in the endometrium. Report of four cases and rewiew of the literature. Am J Clin Pathol 1994; 101: 643-6.

47. Applebaum EL, Frankel A. Extramedullary hematopoiesis of the middle ear. Am J Otolaryngol 1989; 10: 287-90.

48. Ohta Y, Nagashima K, et al. Spinal cord compression due to extramedullary hematopoiesis associated with polycythemia vera-case report. Neurol Med Chir (Tokyo) 2002; 42: 40-3.

49. Martínez-Rodrigo MA, Saldana D, et al. (Spinal cord compression secondary to epidural extramedullary hematopoiesis in thalassemia: A clinical case and review of literature) Rev Neurol 1998; 27: 998-1004. 